\title{
Health workforce cultural competency interventions: a systematic scoping review
}

Crystal Jongen ${ }^{1,2^{*}}$, Janya McCalman ${ }^{1,2}$ and Roxanne Bainbridge ${ }^{1,2}$

\begin{abstract}
Background: Addressing health workforce cultural competence is a common approach to improving health service quality for culturally and ethnically diverse groups. Research evidence in this area is primarily focused on cultural competency training and its effects on practitioners' knowledge, attitudes, skills and behaviour. While improvements in measures of healthcare practitioner cultural competency and other healthcare outcomes have been reported, there are concerns around evidence strength and quality. This scoping review reports on the intervention strategies, outcomes, and measures of included studies with the purpose of informing the implementation and evaluation of future interventions to improve health workforce cultural competence.

Methods: This systematic scoping review was completed as part of a larger systematic literature search conducted on cultural competence intervention evaluations in health care in Canada, the United States, Australia and New Zealand published from 2006 to 2015. Overall, 64 studies on cultural competency interventions were found, with 16 aimed directly at the health workforce.

Results: There was significant heterogeneity in workforce intervention strategies, measures and outcomes reported across studies making comparisons of intervention effects difficult. The two main workforce intervention strategies identified were cultural competency training and other professional development interventions including other training and mentoring. Positive outcomes were commonly reported for improved practitioner knowledge (9/16), skills (7/16), and attitudes/beliefs (5/16). Although health care (6/16) and health (2/16) outcomes were reported in some studies there was very limited evidence of positive intervention impacts. Only four studies utilised existing validated measurement tools to assess intervention outcomes.

Conclusion: Training and development of the health workforce remain a principle strategy towards the goal of improved cultural competence in health services and systems. Diverse approaches are available to increase health workforce cultural competence. However, the effects of interventions beyond practitioner knowledge and attitudes remains unclear. Assessment of practitioner behavioural outcomes as well as measures of intervention impact on healthcare and health outcomes are needed to build a stronger evidence base.
\end{abstract}

Keywords: Cultural competence, Indigenous, Ethnic minorities, Cultural competence training, Health workforce development

\footnotetext{
* Correspondence: crystal.sky.jongen@gmail.com

${ }^{1}$ School of Health, Medicine and Applied Sciences, Central Queensland

University, Cnr Shields and Abbott Streets, Cairns, QLD 4870, Australia

${ }^{2}$ Centre for Indigenous Health Equity Research, Central Queensland

University, Cnr Shields and Abbott Streets, Cairns, QLD 4870, Australia
}

(c) The Author(s). 2018 Open Access This article is distributed under the terms of the Creative Commons Attribution 4.0 International License (http://creativecommons.org/licenses/by/4.0/), which permits unrestricted use, distribution, and reproduction in any medium, provided you give appropriate credit to the original author(s) and the source, provide a link to the Creative Commons license, and indicate if changes were made. The Creative Commons Public Domain Dedication waiver (http://creativecommons.org/publicdomain/zero/1.0/) applies to the data made available in this article, unless otherwise stated. 


\section{Background}

While there is no doubt about the central role of culture in health and health care [1] the concepts of culture, cultural difference and cultural competence are complex and can be difficult to define [2]. Many varied definitions are used to describe cultural competence; one definition commonly used was provided by Cross, Bazron, Dennis and Isaacs (1989) [3]. Cross et al. define cultural competence as "a set of congruent behaviours, attitudes and policies that come together in a system, agency or among professionals that enable that system, agency or professions to work effectively in cross-cultural situations" (p. iv) [3]. This definition accounts for a range of intervention approaches which are used to improve the cultural competence of healthcare systems. One key approach to improving overall health care cultural competence is to develop the capacity of the health workforce to practice in a culturally competent manner.

Health professionals play a key role in determining the nature of interactions and patient experiences when accessing health care. Cultural and linguistic differences between healthcare providers and health service users can results in significant miscommunication [4], as well as service user mistrust [5], decreased satisfaction and disempowerment [6]. In contrast, practitioners' increased cultural competence has been linked to increased patient satisfaction [7, 8], treatment adherence [9] and information seeking and sharing [8]. It is perhaps due to the key role that health practitioners play in determining the health care experiences of patients that improving health workforce cultural competency is one of the oldest and most predominant of cultural competence strategies [10, 11].

The general focus of cultural competence workforce interventions has been on educating and training the health workforce in the requisite and relevant knowledge, attitudes, and skills needed to effectively respond to sociocultural issues arising in clinical encounters [11]. Cultural competence training can include: understanding the central role of culture in all lives and how it shapes behaviour; respect and acceptance of cultural differences; learning to effectively utilise culturally adapted and culturally specific practices; and, continuous development of ones awareness of personal cultural influences and prejudices or biases [12-15]. Cultural competence training has mostly focused on developing knowledge, attitudes, awareness and sensitivity of those working in healthcare. However, the literature reiterates the need to reach further than this, and focus on teaching the skills needed to translate knowledge and awareness into tangible practitioner behaviours which can be consistently applied and assessed in healthcare encounters and settings $[3,10,15,16]$.

Different approaches to cultural competence training have been adopted over the years. Historically, there has been a greater focus on categorical approaches that involve teaching health providers information about particular cultural, ethnic or racial groups. Such approaches describe common health beliefs, attitudes and behaviours of particular groups and offer prescriptive advice about what to do and what not to do in clinical encounters [11]. Nevertheless, it has been acknowledged that categorical approaches are insufficient and problematic for numerous reasons.

To begin with, the categorical approach is critiqued for misrepresenting and oversimplifying the concept of culture as fixed and static [17] rather than a fluid and dynamic phenomenon in a process of constant change and adaptation [16, 18, 19]. Furthermore, the significant cultural, religious, ethnic and national diversity present in many countries means that it is not feasible to be familiar with all cultural perspectives practitioners may encounter $[11,18,20]$. Categorical approaches to cultural competence training may lead to stereotyping which can in fact increase cultural misunderstanding $[11,17,20]$. Such approaches have also been criticised for giving little attention to intra-group variability [19] and for failing to account for the ways in which acculturation and socioeconomic status effect different individuals ways of expressing and experiencing their culture [11].

Another key approach to cultural competence education and training which addresses some of the concerns identified with categorical approaches is the cross-cultural approach. A cross-cultural approach to cultural competence education and training is focused on teaching general knowledge, attitudes and skills relevant to navigating any cross-cultural situation $[11,18]$. Some of these skills and attitudes were outlined by pioneers in cross-cultural medicine such as Berlin and Fowkes [21], Kleinman [22] and Leininger [23], and include: eliciting patients' explanatory models of health issues and their causes; strategies for negotiating shared understanding and facilitating participatory decision-making in creating treatment plans; and understanding health and illness in its biopsychosocial context $[18,20]$. As well as being applicable in clinical encounters with patients from varied cultural and ethnic backgrounds, such approaches have the advantage of being focused on specific skills that can be applied in healthcare encounters.

Cultural competence interventions have come to be considered a key strategy towards addressing racial and ethnic healthcare and health disparities that exist across Canada, Australia, New Zealand, the United States (hereto referred to as the CANZUS nations) [24]. For example, the release of Unequal Treatment in 2002 by the United States (U.S.) Institute of Medicine revealed the critical disparities in the quality of health care received by ethnic and racial minorities [25] and established cultural competence training for healthcare professionals as an important step in addressing these pervasive disparities $[18,26]$. As a result, factors besides cultural differences and cultural barriers came to be included in the discourse and scope of cultural 
competence. These factors include patient mistrust of health practitioners and systems because of historical and contemporary experiences of discrimination and provider bias towards minority groups [20]. Cultural competence training can include developing an awareness of issues of gender, sexuality, and those such as racism, health practitioner and system bias and mistrust $[18,20]$. Critical reflection on practitioner perspectives is also advocated. This includes critically reflecting on and acknowledging the limitations of "medico-centric" frameworks and the effects of dynamics of power and privilege associated with professional status [20].

Positive outcomes have been reported from cultural competency interventions targeting the health workforce, particularly for practitioner outcomes. In their literature review on educational interventions to improve the cultural competence of health care providers, Beach et al. [27] found excellent evidence of improved practitioner knowledge and good evidence of improved practitioner attitudes and skills. However, there is less evidence generally for the impacts of cultural competence education and training interventions on the patient healthcare and health impacts so crucial for determining broader intervention effectiveness. For example, in their review Beach et al. [27] found some evidence for effects of cultural competence education interventions on patient satisfaction. However, poor evidence was found for patient adherence and no health outcomes were reported. Lie et al. [28] reviewed cultural competency workforce interventions that included measures of health outcomes. Although seven studies were found, the studies were of low to moderate methodological quality and showed limited evidence of a positive relationship between cultural competency training initiatives and improved health outcomes.

This paper was developed as part of a broader review of cultural competency interventions in health care for Indigenous peoples and other minority ethnic/cultural groups in the CANZUS Nations [24], the findings of which have been published in our book [2]. The aim of the larger review was to assess the intervention strategies and measures used to increase cultural competence in health care in the CANZUS nations, along with the outcomes reported for these interventions. These countries were selected due to the commonalities in population health and colonial history that exist across these four settler countries $[29,30]$. Several reviews have addressed common issues across the CANZUS nations, reporting particularly on Indigenous health issues [31, 32]. The larger review looks at various cultural competence interventions across multiple healthcare system levels or components. This paper is distinguished from this larger review in that it provides a detailed and evidence-based review of the findings specific to health workforce interventions, a discreet and unique approach to increasing cultural competence in health care.

In this article, we contribute to the existing literature base by providing a systematic scoping review of studies on workforce cultural competency education and training interventions from 2006 to 2015. Its purpose is to inform the implementation and evaluation of future interventions to improve the cultural competence of health professionals. In particular, this review aims to:

- Examine the definitions of culture, cultural difference and cultural competence adopted by the included studies;

- Examine the intervention strategies utilised by studies;

- Report on the measurement approaches taken to evaluate interventions;

- Examine the reported outcomes of included studies.

\section{Methods}

As this review was completed as part of a larger systematic review, details on the inclusion/exclusion criteria, search strategy, identification, screening and inclusion of publications, as well as data extraction and analysis processes used in the broader systematic review have been reported elsewhere [2, 33, 34]. To summarise briefly, the review included peer-reviewed and grey literature published in English from January 1st 2006 to December 31st 2015. Included publications were those which evaluated an intervention designed to improve cultural competence in healthcare for Indigenous or other racial or ethnic minority groups in Australia, Canada, New Zealand or the United States (see Additional file 1 for an overview of the search strategy).

Our comprehensive, six step search strategy and blinded screening process resulted in 64 studies for inclusion in the review (see Fig. 1 for PRISMA search strategy flow chart). Data was extracted for all included studies (see Additional file 2) and the quality of included studies were assessed using the Effective Public Health Practice Project (EPHPP) [35] and Critical Appraisal Skills Programme (CASP) quality assessment tools [36].

The 64 studies found were organised according to the healthcare level or component being addressed. The authors identified four primary cultural competence intervention categories including: health workforce development $(n=16,25 \%)$; student education and training $(n=16,25 \%)$ [37]; programs and services $(n=22,34 \%)$ [33], and; health organisations and systems $(n=10,16 \%)$ [34] (see Fig. 2). The 16 evaluated interventions that aimed to increase cultural competency through health workforce development are reported in this paper. 
SEARCH 1: Jan 2002 - Jul 2012

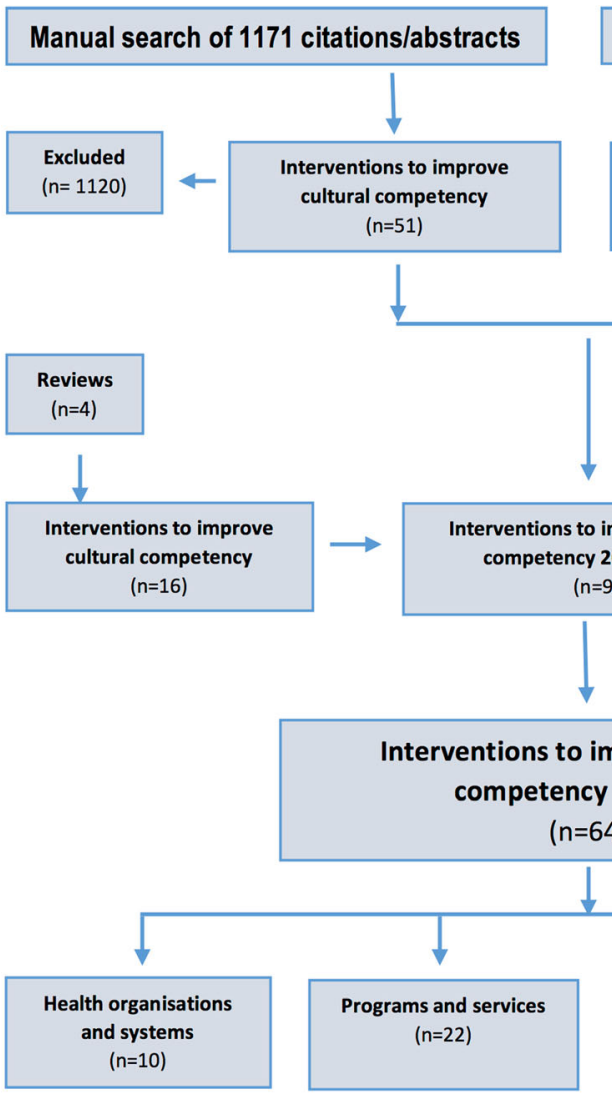

Fig. 1 PRISMA search strategy flow chart

\section{SEARCH 2: Aug 2013 - Dec 2015}

\section{Manual search of 1527 citations/abstracts}
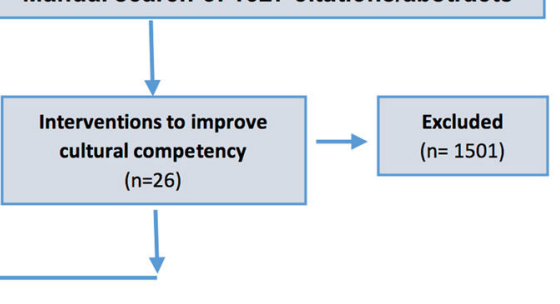

20016 $=93)$

-2006 excluded

$(n=29)$
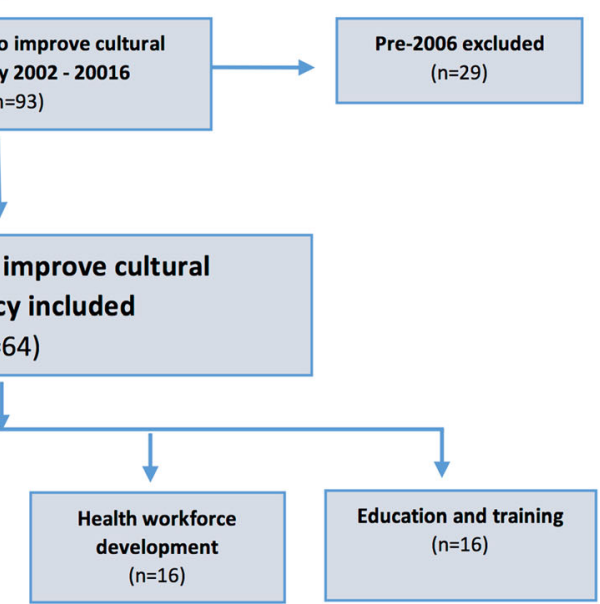

\section{Results}

Definitions of culture, cultural difference and cultural competence

Across all included studies, none provided a definition of culture and only three provided a definition of cultural competence [38-40]. Although sharing some

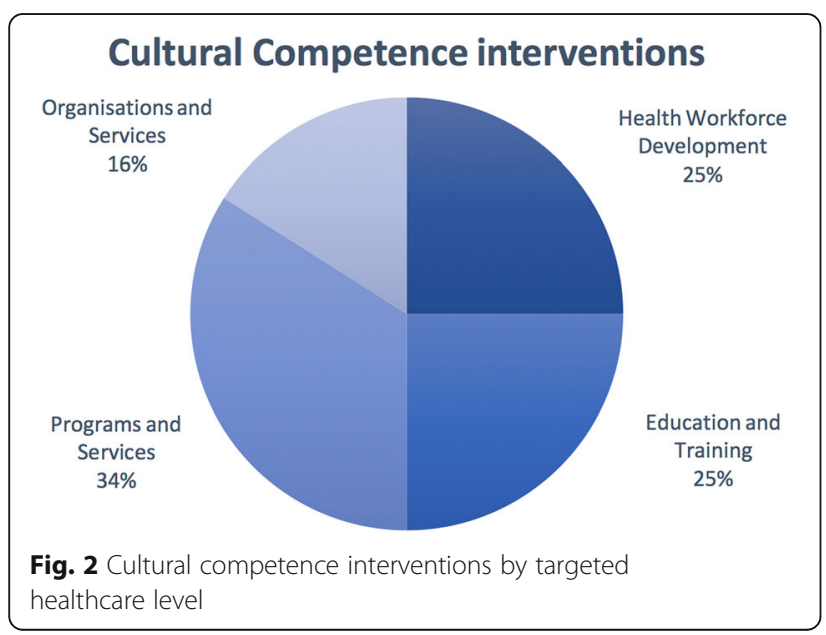

commonalities, all three definitions of cultural competence were distinct. There were also dissimilarities in the primary cultural differences discussed across studies which justified the need for cultural competence. Some included studies discussed cultural differences as distinctions in understandings of health that exist among different population groups, for example the holistic understandings of health held by Indigenous people and how this differs from mainstream approaches to health $[41,42]$. Other studies discussed communication issues related to language discordance and cultural differences and how these affect clinical encounters, particularly for Hispanic populations in the United States [43-45].

\section{Intervention strategies}

Across the included studies, diverse intervention strategies were used to address cultural competence at the workforce level. The two primary workforce strategies were cultural competency training interventions and professional development interventions aimed at improving the cultural competence of health services and practitioners. There was a significant variation in focus, content, mode 
of delivery and duration of interventions within these two primary strategies. There was also heterogeneity in the outcomes reported across the studies. The most common outcomes were for practitioner related cultural competence, along with some healthcare process and health outcomes. See Table 1 for a detailed summary of intervention strategies and outcomes of included studies.

\section{Cultural competence training}

Eleven of the 16 studies reviewed (69\%) provided cultural competence training to the health workforce as the primary intervention. The different approaches to cultural competence training and education discussed previously were reflected in the reviewed studies. Five reported interventions employed a cross-cultural approach focused on teaching broadly applicable knowledge and skills for cultural competence [40, 46-49]. Six interventions utilised a categorical approach, teaching practitioners about certain characteristics, beliefs and behaviours of relevant populations [38, 43-45, 50, 51]. Three studies using categorical approaches focused on Indigenous Australians [38, 50, 51], and three were specific to Latin American peoples [43-45]. Two cultural competency training interventions also included some degree of Spanish language training $[43,45]$. See Table 2 for summaries of cultural competence workforce training approaches.

\section{Professional development interventions}

After cultural competence training, the next most common intervention type was professional development. Professional development interventions used both training and mentoring/supervision to increase the cultural competence of the health workforce. Five studies delivered training concentrated on particular health issues/ fields or specific interventions [41, 42, 48, 51, 52], and four studies used mentoring/supervision strategies [39, 43, 52, 53]. Mentoring and supervision strategies aimed at increasing cultural competency were employed with individual practitioners, entire health services, and minority research faculty and students. See Table 3 for professional development intervention strategies.

\section{Delivery mode}

Another important distinction in the reviewed interventions was in the delivery mode chosen (Table 4 reports on interventions by setting, target group and delivery mode). Only 5/16 studies implemented an intervention in one practice setting. The majority of evaluated interventions $(11 / 16,69 \%)$ were implemented across multiple healthcare services. Studies reported on intervention delivered across multiple sites in one local area [45, 46, 48, $49,52]$, targeting healthcare workers from diverse practice settings $[39,42,51]$, or delivered state or nationwide $[44,47,50]$. Furthermore, there was such variance in the frequency and duration of cultural competency training interventions that an analysis of outcome effects related to course duration cannot be provided.

\section{Study quality}

When assessing for study quality, ten of the 16 studies were found to be weak (63\%), four moderate (25\%) [38, $41,47,50]$ and two strong $[43,49]$.

\section{Intervention outcomes}

The primary outcome measures reported in the reviewed cultural competency workforce development intervention evaluations were health practitioner knowledge (9/16) [38, 40-42, 44-47, 51], attitudes/beliefs (5/16) [38, 45, 48, 51, 52], skills (7/16) [39, 42, 45-47, 51, 52], behaviour (4/16) $[39,49,52,53]$, and confidence $(5 / 16)[39,41,42,48,51]$. Consistent with Beach et al. [27], our results show that many training interventions had positive outcomes independent of whether they were courses of longer or shorter duration. This finding was consistent whether training interventions used a cross-cultural approach to cultural competence training or a categorical approach, with positive outcomes reported across studies .

Although less common, healthcare outcomes such as practitioner satisfaction $(4 / 16)[40,41,51,52]$, patient satisfaction (2/16) [43,49] and patient trust (1/16) [49] were evaluated in some studies. However, only one study reported improved healthcare outcomes in response to a cultural competency intervention, that of increased patient satisfaction [43]. Two studies evaluated the impacts of interventions on patient health outcomes [45, 49], but not significant improvements were reported. The limited evaluation on the health impacts of interventions in the reviewed studies means these results cannot contribute further to the relatively weak evidence-base on the impacts of cultural competence training on patient health outcomes demonstrated by previous research [28]. Three other outcomes reported were increased research productivity, training completion rates, and improved readiness to provide culturally competent care. See Tables 1 and 5 for a summary of outcomes by study.

\section{Measures}

There was significant diversity in the measurement tools used across studies to measure changes in cultural competence, with no studies using the same measurement tool. Seven studies measured practitioner cultural competence using a measurement instrument. Of these seven, four used validated measurement instruments $[38,40,48,52]$. One study used a tool developed by selecting relevant items from existing, validated instruments (reporting a final alpha coefficients for subscales ranging from .70 to .97) [47], and another independently developed a Cultural Competency Assessment (CCA) 


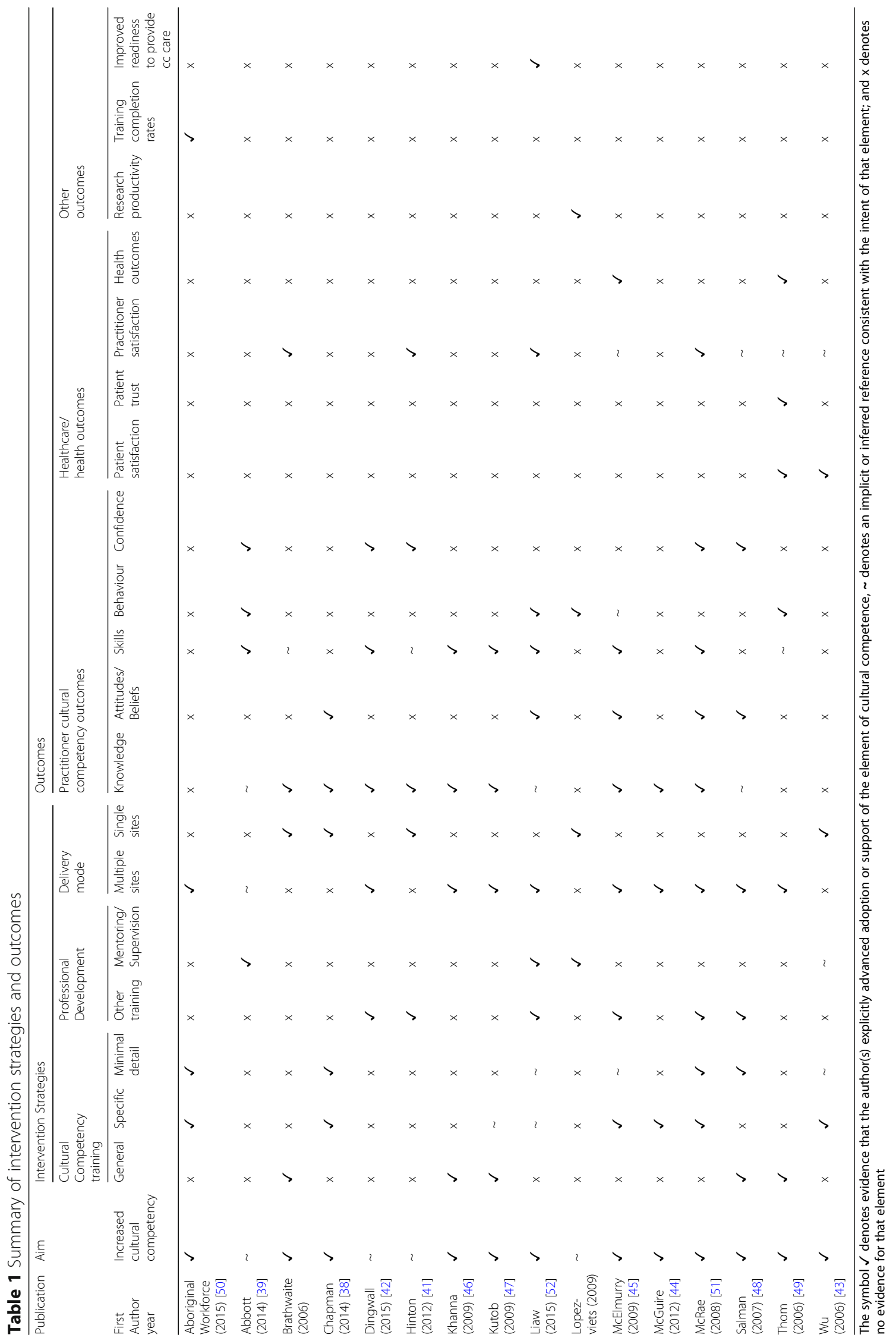


Table 2 Cultural competence training approaches

\begin{tabular}{ll}
\hline Publication & Training approach \\
\hline Cross-cultural approaches \\
Brathwaite & The cultural competency training components of \\
(2006) [40] & cultural awareness; cultural knowledge; cultural \\
& skill; cultural encounter and; cultural desire were \\
& addressed by teaching basic process of providing \\
& culturally competent care such as a) acknowledging \\
& intracultural diversity and the breadth and \\
& complexity of culture as something possessed by \\
& all; b) conducting cultural assessments of service \\
users; c) learning from patients about their culture \\
d) recognizing the processes of acculturation and \\
cultural diversity within individuals; e) developing \\
agreed upon treatment plans; and f) \\
accommodating non-harmful health beliefs and \\
practices which may differ from practitioners \\
personal and professional culture. \\
The training program covered broad cultural \\
competency topics such: as defining cultural and \\
linguistic competency; understanding racial and \\
ethnic health disparities; exploring the relationship \\
between culture and health beliefs; and the \\
importance of cultural competency in effective \\
patient/provider communication. Following training \\
participants were expected to be able to: define \\
culture and describe the spectrum of diversity; \\
understand the differences between ethnicity, race \\
and culture; recognize and define the concepts of \\
intercultural and intracultural diversity; understand \\
the difference between cultural generalizations and \\
stereotypes; define and understand cultural \\
competency; explain the concept of the cultural \\
competency continuum, and; describe the use of \\
explanatory models and their importance in the \\
patient-practitioner interaction.
\end{tabular}

Kutob (2009) Learning objectives included things such as: [47] distinguishing between the terms culture, ethnicity and race; utilizing the Ask, Share, Compare, Negotiate (ASCN) model with patients; describing evidence based information on health disparities as well as health beliefs and behaviours of Mexican American patients; managing potential barriers to blood sugar control for people with type 2 diabetes, and; the appropriate ordering of routine prevention services for diabetes patients. Participants were assessed for things such as their cultural selfawareness, their ability to be open and nonjudgmental, their avoidance of stereotyping, and ability to elicit patients' explanatory model among others.

Salman (2007) Cultural workshop focused on understanding the [48] components of cultural competence and its relevance to healthcare delivery.

Thom (2006) The competencies addressed were knowledge [49] (such as knowledge of cultural identification and levels of agreement with respect to mainstream health beliefs), "communication skills (including listening, explaining, acknowledging, providing recommendations, and working effectively with interpreters); and cultural brokering (including negotiating a treatment plan with patient and family, understanding community resources available to patients, and working with then healthcare system to meet the needs of culturally diverse patients)".

Categorical/Multicultural approaches
Table 2 Cultural competence training approaches (Continued)

\begin{tabular}{|c|c|}
\hline Publication & Training approach \\
\hline $\begin{array}{l}\text { Aboriginal } \\
\text { Workforce } \\
\text { (2015) [50] }\end{array}$ & $\begin{array}{l}\text { Included an eLearning component providing } \\
\text { knowledge on Aboriginal history, culture and } \\
\text { people, and exploring key challenges to providing } \\
\text { culturally appropriate care to Aboriginal people } \\
\text { and communities and a face-to-face workshop } \\
\text { component including both generic content (aimed } \\
\text { at bridging what is learnt in the eLearning } \\
\text { component) and local content (exploring the local } \\
\text { communities being served). }\end{array}$ \\
\hline $\begin{array}{l}\text { Chapman } \\
\text { (2014) [38] }\end{array}$ & $\begin{array}{l}\text { Aimed to provide health practitioners with a } \\
\text { comprehensive understanding of aspects of } \\
\text { Aboriginal culture and ideology. }\end{array}$ \\
\hline $\begin{array}{l}\text { McElmurry } \\
\text { (2009) [45] }\end{array}$ & $\begin{array}{l}\text { Cultural workshops addressed Latino patients' } \\
\text { expectations of care and experiences with health } \\
\text { services, the impacts of cultural beliefs and language } \\
\text { barriers on issues in diagnosis, treatment, and } \\
\text { popular herbal remedies frequently utilised in Latino } \\
\text { cultures. In addition to cultural workshops, this } \\
\text { intervention offered an intensive Spanish language } \\
\text { course, or an integrated immersion program } \\
\text { including Spanish language classes, cultural } \\
\text { workshops, community-based clinical experiences } \\
\text { and home-stays. }\end{array}$ \\
\hline $\begin{array}{l}\text { McGuire (2012) } \\
\text { [44] }\end{array}$ & $\begin{array}{l}\text { A training program focused on factors which may } \\
\text { affect patient-practitioner communication and care } \\
\text { such as: barriers in accessing health care in the U.S.; } \\
\text { differences in health care systems in Latin America } \\
\text { and the U.S.; expectations of Latino patients seeking } \\
\text { care; social and cultural constructs of health and } \\
\text { illness in Latino cultures, and; common health } \\
\text { beliefs and practices such as the use of } \\
\text { complementary medicine. }\end{array}$ \\
\hline $\begin{array}{l}\text { McRae (2008) } \\
\text { [51] }\end{array}$ & $\begin{array}{l}\text { Cultural awareness training workshop regarding } \\
\text { working with Aboriginal Australians. }\end{array}$ \\
\hline Wu (2006) [43] & $\begin{array}{l}\text { Brief training introduced Latino cultural values and } \\
\text { home remedies important to history taking, taught } \\
\text { residents a few Spanish expressions to help establish } \\
\text { rapport with Spanish-speaking patients, and } \\
\text { discussed techniques for optimizing the use of } \\
\text { interpreters in improving communication. }\end{array}$ \\
\hline
\end{tabular}

tool [46]. Lastly, one study measured the knowledge of participants using a pre-post multiple choice questionnaire [44]. The two studies measuring patient satisfaction utilised previously established indicator tools [43, 49] (see Table 5 for further detail on measures).

\section{Discussion}

One of the key issues across the cultural competence literature is the lack of consistent terminology and an agreed upon definition of cultural competence and related concepts [2, 54-56]. As part of this review, we were interested to examine the definitions of culture and cultural competence used across studies as well as the understandings of cultural difference used to justify the need for cultural competence. Across the included studies, there was a general lack of reporting on definitions of cultural competence, culture and cultural difference. The definitions of cultural competence that were 
Table 3 Professional development interventions

\begin{tabular}{lll}
\hline Publication & Other Training & Mentoring/Supervision \\
\hline Abbott (2014) [39] & An intervention to explore (general practitioner) GP Supervisors and \\
& Medical Educators attention to cultural competency when providing \\
& supervision to Medical Registrars. Participants viewed a simulated \\
consultation between an Aboriginal patient and GP Registrar & highlighting inadequacies in communication and cultural awareness \\
and documented teaching points to prioritize in supervision in & \\
response to the video consultation.
\end{tabular}

Dingwall (2015) [42] Evaluated the effects of training in a culturally adapted Indigenous e-mental health application on Indigenous and non- Indigenous service providers' awareness, perceived knowledge and confidence in using the app with Indigenous clients.

Hinton (2012) [41] Evaluated the effects of an Indigenous specific "Yarning about Mental Health" training for the Australian Drug and Alcohol workforce providing culturally appropriate strategies and tools for understanding mental health, promoting wellbeing, and delivering brief, evidencebased interventions.

Liaw (2015) [52] Participating medical practices partook in a cultural respect workshop which provided orientation to the 'Ways of Thinking Ways of Doing' clinical re-design program designed to improve the cultural competency of General Practices.

Viets (2009) [53]

A university-based, culturally centred mentorship program which aimed to train and mentor junior faculty and graduate students from underrepresented backgrounds to conduct addictions-related research projects for Native American, Latino and rural communities and to develop culturally supported interventions (CSI) or adapted empirically supported interventions (ESI) for these communities.

McRae (2008) [51] Pharmacists received training in culturally appropriate teaching strategies then delivered a culturally appropriate program designed to educate Aboriginal Health Workers about cardiovascular medicines management for Aboriginal people.

Salman (2007) [48] Alongside general cultural competency training, this intervention included training in ethno-geriatric care.

Wu (2006) [43]

Individual cultural education sessions were provided to residents where language or cultural issues that emerged during specific clinical encounters were reviewed with the cultural educator. provided in only three of the included studies were all different, confirming the lack of a consistent definition of cultural competence. The use of inconsistent terminology to describe approaches towards the goal of improving cultural competence seen across the literature was also seen in the included studies. Terms used included cultural awareness, cultural respect, cultural safety, cultural understanding, and culturally appropriate healthcare. Furthermore, cultural differences are complex and varied within and across different cultural and ethnic groups [2]. For this reason, it is only logical that the types of differences cultural competency approaches aim to address with be distinct for different population groups.

The interventions reported across the studies included in this review were varied. The primary intervention strategies used were cultural competence training and other professional development activities. Professional development activities included health issue/field or program-specific training, and mentoring/supervision approaches. These interventions represent a diversity of approaches taken to improve health workforce cultural competence across CANZUS nations. This diversity can be considered a strength, demonstrating the many opportunities available to facilitate the ongoing process of improving the cultural competence of the practicing health workforce. However, the heterogeneity of intervention approaches, measures and outcomes makes analysis of interventions and their outcomes more difficult. For this reason, we focus our discussion on the general trends that are seen across studies, particularly those using similar strategies to improve health workforce cultural competence.

Cultural competence training for the health workforce was the most frequently implemented intervention strategy reported across $69 \%$ of the included studies. Cultural 
Table 4 Intervention setting, target group and delivery mode

\begin{tabular}{|c|c|c|}
\hline Publication & Intervention setting and target group & Delivery mode \\
\hline Aboriginal Workforce (2015) [50] & $\begin{array}{l}\text { All health staff in a state based health system, } \\
\text { encompassing primary and secondary health } \\
\text { settings. }\end{array}$ & $\begin{array}{l}\text { State-wide intervention delivered through a 2-h } \\
\text { eLearning component and 6-h face-to-face works }\end{array}$ \\
\hline Abbott (2014) [39] & $\begin{array}{l}\text { Training event for GP Supervisors }(n=71) \text { and } \\
\text { Medical Educators }(n=4) \text {. }\end{array}$ & $\begin{array}{l}\text { Intervention delivered through two non-mandato } \\
\text { Supervisor training days involving practitioners fro } \\
\text { various practice sites. }\end{array}$ \\
\hline Brathwaite (2006) [40] & $\begin{array}{l}\text { Public Health Nurses }(n=75) \text { employed at } \\
\text { a Public Health Department. }\end{array}$ & $\begin{array}{l}\text { The cultural competence program ran two-hour } \\
\text { workshops over five consecutive weeks, with one } \\
\text { booster session at } 1 \text { month. The intervention was } \\
\text { delivered through one organisational site. }\end{array}$ \\
\hline
\end{tabular}

Chapman (2014) [38]

Dingwall (2015) [42]

Hinton (2012) [41]

Khanna (2009) [46]

Kutob (2009) [47]

Liaw (2015) [52]

Lopez-Viets (2009)

McElmurry (2009) [45]

McGuire (2012) [45]

McRae (2008) [51]

Salman (2007) [48]

Thom (2006) [49]

Wu (2006) [43]
Health professionals $(n=63)$.

Emergency Department staff including nursing, clerical and allied health staff $(n=44)$ from in a hospital setting.

Training completed over one-year by health staff of diverse professions $(n=138)$ attending one of ten training courses held in various locations.

Training held for the Alcohol and Other Drug (AOD) workforce $(n=59)$, including AOD workers and counsellors, mental health and allied health workers.

Healthcare providers and administrators $(n=60)$.

Family Medicine Residents ( $n=122)$.

GP clinics $(n=10)$ and their staff.

Ethnic minority junior faculty members at a University $(n=9)$.

Health care professionals and students $(n=386)$ across 5 ambulatory care sites.

Pharmacists $(n=12)$ and Aboriginal Health Workers (AHW) $(n=47)$.

Nursing staff $(n=202)$ in hospital settings.

Primary care physicians ( $n=53)$ across diverse health care practice settings.

Medical residents delivering care to Spanish speaking parents $(n=250)$ in one large teaching hospital.
Three 2-h workshops delivered over 6 weeks to staff at one site.

Multiple health professionals working across the state.

Four 1-day training workshops held over a period of 2 years for members of two AOD workforce network agencies.

$4 \mathrm{~h}$ long cultural competency training delivered to healthcare professionals working across two large regional medical groups.

A 1-h internet-based cultural competency course trialled on a national sample.

Half-day cultural respect workshop, toolkit and cultural mentor to support a clinic re-design process implemented across numerous GP clinics in one region.

A four-year culturally centred mentorship program delivered in one University.

A 3-year demonstration project offering training to health staff across a large regional health service network involving 5 healthcare sites. A six-session cultural workshop series and intensive Spanish language classes were offered as either an 8-week class series or 1-3-week integrated immersion program.

Education DVD delivered to healthcare professionals state-wide through conferences, community meetings and clinic training. A life nationwide webcast and satellite conference was also offered, and the training was accessible online. 26 states were represented in the webcast.

Pharmacists and AHW's based across 10 localities throughout a large regional area. Pharmacists attended an education weekend which included a 4-h cultural awareness session. The health worker education program occurred over 4 sessions ranging from $30 \mathrm{~min}$ to $1.5 \mathrm{~h}$.

Training was provided to nurses working across two major tertiary hospitals.

Intervention was implemented across four diverse health care practice sites. Three modules could be delivered as one half-day training or 3 separate sessions of 1-1.5 $\mathrm{h}$.

30-min group cultural workshop and two individual cultural mentoring sessions delivered to medical residents in one practice setting. competence training interventions were delivered to a range of health professionals. Although some studies reported on training delivered specifically to physicians or nurses, more commonly, cultural competence training was provided to a diverse range of healthcare professionals together. There were no apparent differences in 
Table 5 Study design, measures and outcomes

\begin{tabular}{ll}
\hline Study Design and Measures \\
\hline Abbott (2014) [39] & $\begin{array}{l}\text { Content analysis to determine the type and detail of } \\
\text { the planned feedback, field notes from workshop } \\
\text { discussions and participant evaluations to gain } \\
\text { insight into participant confidence in cross cultural } \\
\text { supervision. }\end{array}$ \\
$\begin{array}{ll}\text { Aboriginal Workforce } \\
\text { (2015) [50] }\end{array}$ & $\begin{array}{l}\text { Data analysis of training completions to measure the } \\
\text { percentage of health staff who completed training } \\
\text { components; staff and participant interviews; and, } \\
\text { web-based survey of Chief Executives. }\end{array}$
\end{tabular}

Brathwaite (2006) [40] Multiple time-series design to measure nurse cultural knowledge as measured by the Cultural Knowledge Scale (CKS).

Chapman (2014) [38] Pre and post questionnaire to measure the cultural awareness (perceptions and attitudes) of staff.

Dingwall (2015) [42] Pre and post questionnaire to measure participant knowledge and confidence in delivering e-mental health to Indigenous people.

Hinton (2012) [41] Pre-post questionnaire to measure participant knowledge and skills.

Khanna (2009) [46] Retrospective post- then pre- evaluation utilising a non-validated Cultural Competency Assessment (CCA) tool to measure changes in knowledge and skills related to the care of patients from diverse cultural and ethnic backgrounds.

Kutob (2009) [47] RCT measuring changes in scores on the Cultural Competence Assessment Tool (CCAT), a self-assessment tool developed for the study.

Liaw (2015) [52]

Lopez-Viets (2009) Pre- and during intervention evaluation in

Pragmatic pre- and post- evaluation using a practice site audit of cultural respect, health checks and risk factor management for Aboriginal patients in general practice. A Cultural Quotient (CQ) questionnaire was used to measure staff cultural strategic thinking, motivation and behaviour. measures of research productivity, including number of grant applications and awards, publications and professional presentations of mentees.

McElmurry (2009) [45] Qualitative written evaluations and pre- and post- program focus groups to measure participants experiences/perceptions, and haemoglobin $A_{1 c}\left(H b A_{1 c}\right)$ levels in patients.

McGuire (2012) [44] Pre-post self-report survey measuring practitioner knowledge and confidence.

McRae (2008) [51] Repeated measures three-phase questionnaire and semi-structured, face-to-face, in-depth interview post-program to evaluate pharmacists confidence. A brief survey to measure acceptability of program to AHWs and an audit of attendance.

Salman (2007) [48]

Pre-post questionnaire to measure practitioner self-reported cultural awareness and competence.

Thom (2006) [49] Randomised Control Trial (RCT) measuring PatientReported Physician Cultural Competence (PRPCC) score, patient satisfaction with and trust in physician, and patient health outcomes of weight, blood pressure and glycosylated haemoglobin.
Outcomes

$72 \%$ registrars referred to culture or to the patient's Aboriginality; few (8\%) documented plans to utilise national initiatives to support health care access for Aboriginal patients. A lack of supervisor confidence in providing guidance on cross-cultural consultation with Aboriginal patients was identified.

Average of 35\% of New South Wales (NSW) Health staff completed online training, with significant variation in completion of face-to-face component across Local Health Districts (LHD). Program implementation was found to be slower than anticipated.

Quantitative and qualitative data showed in an increase in participants cultural knowledge following the program.

Changes in staff perceptions, but not attitudes which remained neutral. A decrease in ambivalence.

Significantly improved perceived knowledge and confidence in using e-mental health tools with Indigenous clients after training.

Significant improvement in knowledge of the warning signs and treatment of mental illness and levels of confidence to assess, treat and communicate with Indigenous mental health clients.

Statistically significant change in participants self-reported knowledge and skills in providing culturally competent care.

Total CCAT scores significantly increased for experimental group participants ( 83.55 before the course to 192.09 after the course), but did not change for the control group.

Practices improved their readiness to provide culturally appropriate care to Aboriginal patients; an increase in Aboriginal patients post intervention $(p<0.05)$.; and increase in cultural quotient score $74.8-89.8(p<0.05)$; and individual practice staff improved their cultural strategic thinking.

There was considerable increase in total mentee research productivity: a 200\% increase in grant applications and awards, a 336\% increase in publications, and a $144 \%$ increase in professional presentations.

Self-reported increased appreciation of cultural interpretations of health, increased knowledge and consideration of Latino health beliefs and practices, improved ability to interact with patients, and greater respect and appreciation for patients cultural views. Improvements in blood glucose control as measured by a drop in $\mathrm{HbA}_{1 c}$.

Significant $(p<0.001)$ improvements in knowledge and confidence.

Significant improvements in confidence with Indigenous health issues and educating AHWs $(p=0.002)$; access to resources to deliver education ( $p=0.005$ ). Education program delivered to $80 \%$ of AHW's in the region with positive reports of participant satisfaction.

No effect sizes reported. Increases in proportion of participants rated as culturally aware and competent.

No significant improvement on any outcome measure for either intervention group. Lack of impact of physician training on health care provision. 
Table 5 Study design, measures and outcomes (Continued)

\begin{tabular}{lll}
\hline Study Design and Measures & Outcomes \\
\hline Wu (2006) [43] & $\begin{array}{l}\text { Comparative study with historical control measuring } \\
\text { parent reported satisfaction with interpreter and }\end{array}$ & $\begin{array}{l}\text { Use of an in-person interpreter significantly increased Latino parents } \\
\text { satisfaction }(p<0.001) \text { versus phone interpreter, but a program using } \\
\text { healthcare experience. }\end{array}$ \\
& $\begin{array}{l}\text { an interpreter to educate residents in cultural and language issues } \\
\text { increased parents' satisfaction more. }\end{array}$ \\
\hline
\end{tabular}

the training delivered to specific or mixed healthcare professionals in the strategies or outcomes reported. This indicates that many cultural competence training interventions are quite generic in nature, and do not necessarily target specific skills and knowledge, or types of care relationships that exist in health care.

There were many commonalities across cultural competency training intervention strategies and outcomes. These commonalities help to shed light on some key strengths and limitations of common approaches to cultural competence training. For example, out of the eleven studies evaluating cultural competency training interventions, six utilised a categorical approach and five implemented a cross-cultural approach. Interventions using either categorical or cross-cultural approaches reported positive outcomes around practitioner knowledge, attitudes/beliefs and reported skills and confidence. Due to the heterogeneity in measurement instruments and assessment methods, we were unable to discern whether either of these approaches to cultural competence training had a greater impact on particular learning outcomes. Despite this, there are some important issues pertaining to these approaches.

As discussed in the introduction, categorical approaches to cultural competence training can run the risk of increasing cultural misunderstanding if they do not account for inter-group variability. There are however certain instances in which categorical cultural competence training approaches can be effective or appropriate [18]. For example, if the cultural competence training is teaching about cultures of local-level populations with the help of local cultural experts, this can help to build cultural competence. Two of the included studies using categorical approaches included a focus on local level populations in line with this recommendation $[44,50]$. The three remaining categorical cultural competence training studies either did not utilise such an approach, or did not report it. Another instance in which categorical approaches may be appropriate is when knowledge which has a clear, evidence-based effect on health care delivery or patient outcomes is being taught. Only one study utilising a cross-cultural approach to cultural competence training mentioned teaching such evidence-based knowledge [47]. Aside from these instances, to avoid generalisations which may lead to cultural misunderstanding, a more suitable tactic is to learn as much as possible directly from patients about their own sociocultural perspectives and how they see this impacting their encounters with healthcare practitioners [18].

Processes and skills for learning directly from patients is something that is commonly addressed in crosscultural education models which have been established to inform the training of health professionals in culturally competent care [57]. For example, Kleinman's explanatory model of disease [22] is a tool which can be used to facilitate cross-cultural communication, increasing understanding between patients and providers by eliciting patients own explanation of their health and or/ illness. This tool is designed to help health providers better understand people's health beliefs, personal and social meanings attached to health issues, and expectations about the therapeutic process [22]. Another key model for cross-cultural education is the LEARN (Listen, Explain, Acknowledge, Recommend, Negotiate) model developed by Berlin and Fowkes (1983) [21]. The LEARN model focuses on teaching generic skills for communication and negotiation that can be applied across all interactions when negotiating difference (cultural or otherwise) in the patient-practitioner encounter. Yet despite the existence and use of these models for many years, only two studies reviewed [47, 49] identified utilisation of both the LEARN model and Kleinman's explanatory model.

To establish the relative impacts of different approaches to cultural competence training, comparative evaluations of interventions are needed to assess impacts using the same measurement instruments. Given the level of heterogeneity in cultural competency training interventions, a tool to assess the themes, concepts, methods and learning objectives of training interventions, such as the one utilised by Dolhun, Muñoz and Grumbach [58] would contribute greatly towards the comparison of outcomes between interventions. To facilitate greater analysis and comparison of cultural competency training approaches, it is important that evaluations provide sufficient detail on training approaches and content. This kind of detail was something that was lacking in many of the cultural competence training studies reviewed. Four of the cultural competence training studies using categorical approaches [38, $43,50,51$ ] and one using a cross-cultural approach [48] did not provide sufficient information to clearly ascertain the content and focus of the training. 
Despite the importance of issues of racism, discrimination and practitioner bias as issues to be addressed in culturally competent health care, none of the reviewed studies evaluating cultural competence training interventions explicitly discussed issues of racism and practitioner bias or stated that these issues were addressed in cultural competence training. Only one intervention evaluated addressed distinguishing between cultural generalisations and stereotypes in a cultural competence training program [46]. The lack of attention to issues of racism and bias is consistent with the findings of other cultural competence reviews. Beach et al. [27], for example, found only two of 34 studies which mentioned concepts of bias, racism or discrimination . Truong et al. (2014) found that although these issues were noted in some cultural competence literature reviews, they were not addressed as outcomes measures [54]. Considering the impact of issues such as racism and practitioner bias on healthcare disparities $[2,25,59]$ interventions to improve health workforce cultural competence should address these issues and include them in outcome evaluations.

This review found two types of professional development strategies reported in included studies that have not been commonly reported in cultural competence literature reviews. One such strategy was training interventions other than cultural competence training which also aimed to increase healthcare workforce cultural competence. These training approaches included training regarding specific health issues/fields (eg. Indigenous mental health and wellbeing [41] and ethno-geriatric care [48]) and training in particular service-level interventions (eg. A culturally adapted Indigenous e-mental health mobile phone application [42], culturally appropriate teaching strategies for training Aboriginal Health Workers [51], and orientation to a cultural respect clinical re-design program [52]). It is recommended that a whole of organisation approach is taken where efforts to improve cultural competence are integrated into all professional development endeavours within a healthcare service [60]. These types of health issue or program specific training interventions are one strategy towards this goal. These training interventions demonstrate the different ways in which efforts to increase health professional cultural competence can be integrated into diverse professional development initiatives.

Mentoring and supervision was another strategy found $[39,43,52,53]$, with studies demonstrating the versatility and potential of this approach as a cultural competency workforce development strategy. Mentoring is a common and effective approach towards personal and career development in the workplace [61]. Mentoring relationships are focused on mentee's learning and encourage a reflective dynamic where openness to feedback is embraced [62]; hence mentoring strategies could encourage the kind of life-long learning processes needed to continuously strive towards cultural competence. A significant focus of literature on mentoring and supervision in the context of cultural competence in the health workforce has been regarding supervision for minority practitioners by Caucasian supervisors [62-66]. However, there are also examples of research which explores the value of cultural mentoring in promoting the cultural competence of doctors working with Aboriginal and Torres Strait Islanders [67]. The potential of mentoring and supervision approaches to improve health practitioner cultural competence is a a research area worth further exploration and testing for its efficacy and impact.

This review did not purposely search for intervention studies aiming to improve health practitioner linguistic competence. However, considering the evidence demonstrating the impact of language discordance on patient satisfaction and quality of care $[68,69]$, the relative absence of efforts to address linguistic differences in most cultural competence workforce development interventions is discouraging. One of the reviewed studies provided Spanish language courses and an immersion program for health practitioners [45]. Another intervention focused on evaluating the effectiveness of interpreter services taught medical residents the use of Spanish expressions within a cultural education training program [43]. This lack of training to address language discordance in cultural competence training is consistent with previous research. Dolhun, Muñoz and Grumbach (2003) found that medical schools rarely addressed language issues in cultural competency course content, such as through teaching about the use of interpreters [58]. Interventions to improve cultural competence in the health workforce in CANZUS nations ought to address linguistic competence as a core aspect of cultural competence, particularly for populations who do not speak English as a first language.

The included studies report some positive effects of workforce cultural competence interventions, particularly on health professionals' knowledge, skills and attitudes or beliefs. However, they did not report on behavioural outcomes of cultural competence interventions. One study assessed patient-reported physician cultural competence behaviours but found no changes following the intervention [49]. The only behavioural changes reported in studies included increased research productivity [53] and physical changes in practice settings to be more culturally sensitive [52]. Hence, the majority of cultural competence training for the health workforce remains focused on building awareness and associated changes in attitudes [60]. However, knowledge and attitude-based outcomes are not sufficient to demonstrate practitioner cultural competence. In order to build a stronger evidence base on the impact of cultural competency workforce interventions it is important that evaluations include assessment of practitioner behavioural outcomes. Cultural competence training approaches should prioritise the teaching of practical skills and the 
application of these skills in practice, as well as their assessment through demonstrable practitioner behaviour $[12,16$, 57]. Assessment of behavioural outcomes could also contribute to evaluation of workforce cultural competence training impacts on patient healthcare and health outcomes. The assessment of healthcare and health outcomes are very important if we hope to demonstrate that cultural competence interventions do in fact impact on the healthcare disparities so frequently used to justify cultural competence interventions [2]. However, only two of the reviewed studies reported healthcare outcomes with one reporting improvements in patient satisfaction [43] and the other reporting no effect [49]. Health outcomes were assessed in two studies $[45,49]$ however neither reported significant changes as a result of interventions impacts. As long ago as 2003, Betancourt outlined a potential approach to cultural competence education and training intervention evaluation which assesses behavioural outcomes related to knowledge and skills taught and their impact on healthcare and health outcomes [18]. Cultural competence training and other workforce development interventions would greatly benefit from applying such an evaluation approach.

There are several key issues in the measurement and evaluation of cultural competence training and workforce development interventions which have been identified in previous literature and are mirrored in the studies reviewed. One concern is the lack of consistency in measurement instruments used to assess intervention outcomes, especially among cultural competency training evaluations. Similar to previous research $[27,70]$ we found no studies using the same assessment tool and little uniformity across studies in measurement of outcomes, even within the same outcome categories. This lack of consistency in measurement tools makes it difficult to compare intervention outcomes and effectiveness across studies.

The over-reliance of self-report measures is an ongoing limitation and concern across the cultural competency literature [14, 54, 70]. Self-report measures were the most common method of evaluation, utilised in $69 \%$ of included studies. However self-report measures are highly subjective and cannot be seen as predictive of resulting behaviour in clinical encounters [18]. Due to the effects of socialdesirability bias, participants might select responses seen as socially appropriate but which are not reflective of their true beliefs $[18,70,71]$. To improve the evidence supporting their effectiveness, interventions aimed at improving health practitioner cultural competence need to move beyond the reliance on self-assessmen measures [54].

Patient assessed practitioner cultural competence is one potential approach to evaluating the impact of cultural competency training interventions which could be used instead of, or in addition to, practitioner self-assessment. Patient assessed practitioner cultural competence has been associated with improved healthcare and health outcomes [72, 73], however there is less evidence linking patient-assessed practitioner cultural competence and associated positive outcomes to impacts of cultural competence training. In the reviewed studies, only one assessed patient reported physician cultural competence behaviours and its correlation with patient satisfaction and trust with no impacts reported [49]. To increase the objectivity of the evidence base for the impact of cultural competency interventions, consistent assessment of patient perceived practitioner cultural competence, as well as healthcare and health outcomes, are needed.

\section{Limitations}

The publications reviewed were identified using a search strategy including electronic databases, websites/clearinghouses and reference lists of reviews designed to discover peer and non-peer reviewed publications that evaluated health service cultural competency interventions. Therefore, it is highly likely that the studies in this review are representative of published cultural competence research from CANZUS nations. However, being a non-exhaustive search strategy it is possible some relevant publications were not found. Furthermore, considering the relative paucity of published studies found in this search, despite the inclusion of studies across four countries in a 10 year time-frame, the few studies found may suggest that many interventions are either not evaluated or not published. The reliance on published peer-reviewed and grey literature is therefore another limitation of this review.

The heterogeneity of included research aims, interventions and outcomes in the included studies is a further limitation. Although this heterogeneity is useful for demonstrating the diversity of approaches which can be taken to improve cultural competence in healthcare, it makes it difficult to draw firm conclusions about the nature of interventions and their associated outcomes. Additionally, because of the breadth and complexity of cultural competence, this review only included studies which explicitly addressed improving cultural competence as an aim of interventions; this possibly excluded studies which implicitly aimed to increase cultural competence. For example, our search did not produce any studies on the recruitment and retention of minority staff as a workforce development, cultural competence strategy.

\section{Conclusion}

The studies which informed this review demonstrate a great diversity in approaches taken to address the cultural competence of the health workforce. Research exploring the comparative benefits of different approaches to cultural competence training as well as the benefits of other professional development opportunities such as mentoring and supervision would be of value to advance knowledge in this area. Although several positive outcomes were reported 
across the included studies, consistent evaluation approaches are needed to build the evidence base on intervention impacts. In particular, greater focus is needed on evaluating the application of knowledge, attitudes and skills in practice and the impacts of cultural competence interventions on specific practitioner behaviours and their subsequent impact on healthcare and health outcomes.

\section{Additional files}

Additional file 1: Searches 1 and 2. Search summary for searches 1 and 2. This figure provides a summary of the literature searches undertaken to inform this review. The search summary details the peer-reviewed electronic databases as well as websites and clearinghouses searches, along with the search terms used. (JPEG $1745 \mathrm{~kb}$ )

Additional file 2: Data extraction table. Cultural competence workforce interventions data extraction table. This table provides detail on the data extracted from reviewed studies including: the author, year and publication type; the country and population; participants and health care setting; intervention type; study design; outcome measure or indicator; assessed outcomes; and study quality. (DOCX 108 kb)

\section{Abbreviations}

AHW: Aboriginal health workers; AOD: Alcohol and other drug; ASCN: Ask, share, compare, negotiate; CANZUS: Canada, Australia, New Zealand, United States; CASP: Critical appraisal skills programme; CCA: Cultural competency assessment; CCAT: Cultural competence assessment Tool; CKS: Cultural knowledge scale; CQ: Cultural quotient; CQU: Central Queensland University; CSI: Culturally supported interventions; EPHPP: Effective public health practice project; ESI: Empirically supported interventions; GP: General practitioner; $\mathrm{HbA}_{1 c}$ : Hemoglobin $\mathrm{A}_{1 c}$; LEARN: Listen, explain, acknowledge, recommend, negotiate; LHD: Local health districts; NSW: New South Wales; PRISMA: Preferred reporting items for systematic reviews and meta-analyses; PRPCC: Patient-reported physician cultural competence; RCT: Randomised control trial; U.S.: United States

\section{Acknowledgements}

The authors acknowledge the contributions of Mary Kumvaj (MK) in conducting the literature search, Komla Tsey (KT) for participating in the screening process, and Anton Clifford (AC) for participating in the screening process and contributing authorship to the larger systematic review which this review is part of.

\section{Funding}

JM and RB have permanent positions with Central Queensland University (CQU). Each of their contributions to the project were completed as part of their established roles with the university. The CQU research support funds of $J M$ and RB were used to further support this project. These funds were used hire a qualified librarian (MK) to complete the search. CQU research support funds also provided the salary of CJ to complete the data analyses and interpretation, and to write the manuscript.

\section{Availability of data and materials}

All data generated or analysed during this study are included in this published article and its supplementary information files.

\section{Authors' contributions}

$\mathrm{CJ}$ is the primary author and was responsible for the data extraction of the search update and the writing of the final review manuscript. RB completed the data extraction for the first search and authored a paper on the larger review in 2014 which informed this review. JM, RB and CJ all contributed significantly towards the development of the review concept and structure, and were involved in drafting of the paper and critically reviewing content during the editing process. CJ, JM and RB have all previewed the final version of the review and have approved it for publication. All authors are assured of the accuracy and integrity of the review and agree to be accountable for all aspects of the publication manuscript.
Ethics approval and consent to participate

Not applicable.

\section{Consent for publication}

Not applicable.

\section{Competing interests}

The authors declare that they have no competing interests and that the research was conducted in the absence of any commercial or financial relationships that could be construed as a potential conflict of interest.

\section{Publisher's Note}

Springer Nature remains neutral with regard to jurisdictional claims in published maps and institutional affiliations.

Received: 30 December 2016 Accepted: 14 March 2018

Published online: 02 April 2018

\section{References}

1. Napier AD, et al. Culture and health. Lancet. 2014;384(9954):1607-39.

2. Jongen C, McCalman J, Bainbridge R, Clifford A. Cultural competence in health: a review of the evidence. Singapore: Springer Nature; 2018.

3. Cross TL, et al. Towards a culturally competent system of care: a monograph on effective services for MinorityChildren who are severely emotionally disturbed. Washington, DC: Georgetown University, Child Development Center; 1989.

4. Cass A, et al. Sharing the true stories: improving communication between aboriginal patients and health care workers. Med J Aust. 2002;176(10):466.

5. Shahid S, Finn L, Thompson S. Barriers to participation of aboriginal people in cancer care: communication in the hospital setting. Med J Aust. 2009; 190(10):574.

6. Roe YL, Zeitz CJ, Fredericks B. Study protocol: establishing good relationships between patients and health care providers while providing cardiac care. Exploring how patient-clinician engagement contributes to health disparities between indigenous and non-indigenous Australians in South Australia. BMC Health Serv Res. 2012;12(1):1-10.

7. Castro A, Ruiz E. The effects of nurse practitioner cultural competence on Latina patient satisfaction. J Am Acad Nurse Pract. 2009;21(5):278-86.

8. Paez KA, et al. Physician cultural competence and catient ratings of the patient-physician relationship. J Gen Intern Med. 2009;24(4):495-8.

9. Roncoroni J, et al. Patient perceived cultural sensitivity of clinic environment and its association with patient satisfaction with care and treatment adherence. Am J Lifestyle Med. 2014;8(6):421-9.

10. Brach C, Fraserirector I. Can cultural competency reduce racial and ethnic health disparities? A review and conceptual model. Med Care Res Rev. 2000; 57(Suppl 1(4)):181-217.

11. Betancourt JR, et al. Defining cultural competence: a practical framework for addressing racial/ethnic disparities in health and health care. Public Health Rep. 2003;118(4):293-302.

12. Campinha-Bacote J. A model and instrument for addressing cultural competence in health care. J Nurs Educ. 1999;38(5):203.

13. Warren BJ. The interlocking paradigm of cultural competence: a best practice approach. J Am Psychiatr Nurses Assoc. 2002;8(6):209-13.

14. Purnell LD. Transcultural Health Care: A Culturally Competent Approach. Vol. 4. Philadelphia: F. A. Davis Company; 2012.

15. Hark L, DeLisser H, Morrison G. Achieving Cultural Competency: A CaseBased Approach to Training Health Professionals. Vol. 1. Chichester, UK: Wiley-Blackwell; 2009.

16. Dreachslin JL, Gilbert MJ, Malone B. Diversity and Cultural Competence in Health Care: A Systems Approach. Vol. 1. New York: Wiley; 2012.

17. Gregg J, Saha S. Losing culture on the way to competence: the use and misuse of culture in medical education. Acad Med. 2006;81(6):542-7.

18. Betancourt JR. Cross-cultural medical education: conceptual approaches and frameworks for evaluation. Acad Med. 2003;78(6):560-9.

19. Thackrah RD, Thompson SC. Confronting uncomfortable truths: receptivity and resistance to aboriginal content in midwifery education. Contemp Nurse. 2013;46(1):113-22

20. Saha S, Beach MC, Cooper LA. Patient centeredness, cultural competence and healthcare quality. J Natl Med Assoc. 2008;100(11):1275-85.

21. Berlin EA, Fowkes JWC. A teaching framework for cross-cultural health care. Application in family practice. West J Med. 1983;139(6):934-8. 
22. Hark L, DeLisser H. Appendix 2: Kleinman's explanatory model of illness. Oxford, UK: Wiley-Blackwell; 2009. p. 217-9.

23. Leininger MM, McFarland MR. Transcultural Nursing: Concepts, Theories, Research and Practice. Vol. 3. New York: McGraw-Hill, Medical Pub. Division; 2002.

24. Meyer WH. Indigenous rights, global governance, and state sovereignty. Hum Rights Rev. 2012;13(3):327-47.

25. Smedley $\mathrm{BD}$, et al. Unequal treatment: confronting racial and ethnic disparities in health care. Washington, D.C: National Academies Press; 2003

26. Williams DR, Rucker TD. Understanding and addressing racial disparities in health care. Health Care Financ Rev. 2000;21(4):75.

27. Beach MC, et al. Cultural competency: a systematic review of health care provider educational interventions. Med Care. 2005;43(4):356-73.

28. Lie DA, et al. Does cultural competency training of health professionals improve patient outcomes? A systematic review and proposed algorithm for future research. J Gen Intern Med. 2011;26(3):317-25.

29. Ford L, Rowse T. Between indigenous and settler governance. New York: Taylor and Francis; 2012.

30. Smylie, J. and P. Adomako, Indigenous Children's Health Report. 2009, Centre for Research on Inner City health: Toronto, Canada.

31. Gould GS, et al. Should anti-tobacco media messages be culturally targeted for indigenous populations? A systematic review and narrative synthesis. Tob Control. 2013;22(4):1-10.

32. McCalman J, et al. Family-centred interventions for indigenous early childhood well-being by primary healthcare services. Cochrane Database Syst Rev. 2016;12:1-18.

33. Jongen CS, McCalman J, Bainbridge RG. The implementation and evaluation of health promotion services and programs to improve cultural competency: a systematic scoping review. Front Public Health. 2017;5(24):1-14.

34. McCalman J, Jongen C, Bainbridge R. Organisational systems approaches to improving cultural competence in healthcare: a systematic scoping review of the literature. Int J Equity Health. 2017;16(78):1-19.

35. National Collaborating Centre for Methods and Tools. Quality assessment tool for quantitative studies. Hamilton, ON: McMaster University; 2008.

36. CASP, Critical Appraisal Skills Programme (CASP) Qualitative Research Checklist. Oxford: 2015. http://www.casp-uk.net/. Accessed 23 Mar 2016.

37. Clifford A, et al. Cultural competency training and education in the universitybased professional training of health professionals: characteristics, quality and outcomes of evaluations. Divers Equality Health Care. 2017;14(3):136-47.

38. Chapman R, Martin C, Smith T. Evaluation of staff cultural awareness before and after attending cultural awareness training in an Australian emergency department. Int Emerg Nurs. 2014;22(4):179-84.

39. Abbott $\mathrm{P}$, et al. General practitioner supervisor assessment and teaching of registrars consulting with aboriginal patients - is cultural competence adequately considered? BMC Med Educ. 2014;14(1):1-8.

40. Brathwaite AC, Majumdar B. Evaluation of a cultural competence educational programme. J Adv Nurs. 2006;53(4):470-9.

41. Hinton R, Nagel T. Evaluation of a culturally adapted training in indigenous mental health and wellbeing for the alcohol and other drug workforce. ISRN Public Health. 2012;2012:1-6.

42. Dingwall KM, et al. Evaluation of a culturally adapted training course in indigenous e-mental health. Australas Psychiatry. 2015;23(6):630.

43. Wu AC, et al. The interpreter as cultural educator of residents. Arch Pediatr Adolesc Med. 2006;160(11):1145.

44. McGuire AA, Garcés-Palacio IC, Scarinci IC. A successful guide in understanding Latino immigrant patients: an aid for health care professionals. Fam Community Health. 2012;35(1):76.

45. McElmurry BJ, et al. Implementation, outcomes, and lessons learned from a collaborative primary health care program to improve diabetes care among urban Latino populations. Health Promot Pract. 2009;10(2):293-302.

46. Khanna SK, Cheyney M, Engle M. Cultural competency in health care: evaluating the outcomes of a cultural competency training among health care professionals. J Natl Med Assoc. 2009;101(9):886-92.

47. Kutob RM, Senf JH, Harris JJM. Teaching culturally effective diabetes care: results of a randomized controlled trial. Fam Med. 2009;41(3):167.

48. Salman A, et al. Cultural competence among staff nurses who participated in a family-centered geriatric care program. J Nurses Staff Dev. 2007;23(3):103-11.

49. Thom DH, et al. Development and evaluation of a cultural competency training curriculum. BMC Med Educ. 2006;6(1):1-9.

50. Aboriginal Workforce. Respecting the Difference: An Aboriginal Cultural Training Framework for NSW Health - Process Implementation Evaluation Report 2013. Sydney: NSW Ministry of Health; 2015.
51. McRae M, et al. Evaluation of a pharmacist-led, medicines education program for aboriginal health workers. Rural Remote Health. 2008;8(4):946

52. Liaw S-T, et al. Improving cultural respect to improve aboriginal health in general practice: a multi-methods and multi-perspective pragmatic study. Aust Fam Physician. 2015;44(6):387-92.

53. Viets $V L$, et al. Reducing health disparities through a culturally centered mentorship program for minority faculty: the southwest addictions research group (SARG) experience. Acad Med. 2009;84(8):1118.

54. Truong M, Paradies Y, Priest N. Interventions to improve cultural competency in healthcare: a systematic review of reviews. BMC Health Serv Res. 2014;14(1):99.

55. Grant J, Parry Y, Guerin P. An investigation of culturally competent terminology in healthcare policy finds ambiguity and lack of definition. Aust N Z J Public Health. 2013;37(3):250-6.

56. Foronda CL. A concept analysis of cultural sensitivity. J Transcult Nurs. 2008; 19(3):207-12.

57. Suh EE. The model of cultural competence through an evolutionary concept analysis. J Transcult Nurs. 2004;15(2):93-102.

58. Dolhun EP, Munoz C, Grumbach K. Cross-cultural education in U.S. medical schools: development of an assessment tool. Acad Med. 2003;78(6):615-22.

59. Fiscella K, Sanders MR. Racial and ethnic disparities in the quality of health care. Annu Rev Public Health. 2016;37:375-94.

60. Curtis EF, Dreachslin $J$, Sinioris M. Diversity and cultural competence training in health care organizations: hallmarks of success. Health Care Manag. 2007;26(3):255.

61. Allen TD, Poteet ML. Enhancing our knowledge of mentoring with a person-centric approach. Ind Organ Psychol. 2011;4(1):126-30.

62. Wong LCI, Wong PTP, Ishiyama FI. What helps and what hinders in cross-cultural clinical supervision: a critical incident study. Couns Psychol. 2013;41(1):66-85.

63. Hird JS, et al. Visions and realities: supervisee perspectives of multicultural supervision. J Multicultural Couns Dev. 2001;29(2):114-30.

64. Falender CA, Burnes TR, Ellis MV. Multicultural clinical supervision and benchmarks: empirical support informing practice and supervisor training. Couns Psychol. 2013;41(1):8-27.

65. Crutcher BN. Cross-cultural mentoring: a pathway to making excellence inclusive. Lib Educ. 2014;100(2):26.

66. Johnson-Bailey J, Cervero RM. Mentoring in black and white: the intricacies of cross-cultural mentoring. Mentoring Tutoring: Partnership Learning. 2004;12(1):7-21.

67. Reath J, et al. Building aboriginal and Torres Strait islander cultural education and cultural mentoring capacity. Sydney: University of Western Sydney; 2013.

68. Morales LS, et al. Are latinos less satisfied with communication by health care providers? J Gen Intern Med. 1999;14(7):409-17.

69. Carrasquillo $\mathrm{O}$, et al. Impact of language barriers on patient satisfaction in an emergency department. J Gen Intern Med. 1999;14(2):82-7.

70. Kumaş-Tan Z, et al. Measures of cultural competence: examining hidden assumptions. Acad Med. 2007;82(6):548-57.

71. Geron SM. Cultural competency: how is it measured? Does it make a difference? Generations. 2002;26(3):39-45.

72. Gaston GB. African-Americans' perceptions of health care provider cultural competence that promote HIV medical self-care and antiretroviral medication adherence. AIDS Care. 2013;25(9):1159-65.

73. Michalopoulou $\mathrm{G}$, et al. Linking cultural competence to functional life outcomes in mental health care settings. J Natl Med Assoc. 2014;106(1):42-9.

\section{Submit your next manuscript to BioMed Central and we will help you at every step:}

- We accept pre-submission inquiries

- Our selector tool helps you to find the most relevant journal

- We provide round the clock customer support

- Convenient online submission

- Thorough peer review

- Inclusion in PubMed and all major indexing services

- Maximum visibility for your research

Submit your manuscript at www.biomedcentral.com/submit 